\title{
A Fiction on Image Retrieval in Dermatology
}

\author{
Sudhakar Putheti, Krishna Prasad Palli, Mohan Krishna Kotha
}

\begin{abstract}
:on this paper we proposed, a novel structure to help and mechanize the gauge of diseases from PC based totally photograph examination methodology the utilization of substance based by and large photo recovery (cbir).cbir is the device of recovering related photographs from huge database accumulations by strategy for technique for utilizing low degree photo highlights including shading, surface and shape and packs of others. we have utilized comfortable texton and discrete shearlet move up to clear surface and structure abilties. the fact of the matter is to manual medicinal fundamental authority, the pass on of a hard and fast dataset which merges pix of a tremendous kind of past pathologically-affirmed events from great assortments of diseases dealing a particular organ which might be shown to be of over the top closeness to the ones of different new obscure occasions basic leadership by techniques for the usage of recovering and showing up past occasions apparently like the handiest under test with pertinence comments utilizing help vector machines.
\end{abstract}

Keywords:Content Based Medical Image Retrieval, Fuzzy Texton, Texels, and Support Vector Machines.

\section{INTRODUCTION}

maximum via an extended shot of the work in dermatology has concentrated on pores and skin perilous advancement revelation. special manner for department, spotlight extraction and social affair have been spoken to with the resource of some creators. regarding department, celebi et al.5presented an intentional unfold angle on late fringe area strategies: amassing looked for after with the aid of thrilling shapes are the most widespread. diverse highlights have been expelled from pores and skin pix, along with form, shading, floor and fringe residences 6 , eight. depiction structures extend from discriminant examination to neural systems and give a boost to vector machines 9,10 . the ones systems are on a truely important dimension conveyed for images gotten by means of manner of epiluminescence microscopy(elm or dermoscopy) and they rotate around cancer, which is extremely a as an alternative terrific, at any charge astoundingly risky, situation except different pores and skin risky improvements are basically step by step regular.

due to the fact the instigating of video and picture information within the the front line form has prolonged, content primarily based photo retrieval (cbir) has modified into an simple studies trouble. as such a tremendous hassle that must be tended to is expedient restoration of images from expansive databases. to find out images which is probably perceptually like a solicitation picture, picture

Revised Manuscript Received on April 12, 2019.

Dr. SudhakarPutheti,VVIT, Nambur, (Email: sudhakarp0101@gmail.com Krishna Prasad Palli,VVIT, Nambur, (Email: krishnaprasad.palli@gmail.com)

Mohan Krishna Kotha,VVIT, Nambur, (Email: mohankrishnakotha@gmail.com) restoration frameworks try to search for through a database. cbir may want to altogether be able to replace the exactness of the records being decrease returned and is an important choice and supplement to ordinary substance based totally photograph pursuing. for delineating image content material, shading, surface and form highlights have been utilized. shading is a victor maximum of the maximum widely utilized low-diploma seen highlights and is invariant to image length and advent 1 . there are shading histogram, shading correlogram as widespread shading highlights carried out in cbir. with out a different statistics, specific articles in a photograph may be visible completely through their surfaces. ground can also portray the critical exercise plan of a region and the relationship of the consolidating zones and may in like manner consist of a few maximum vital close by people. shape be a part of has been comprehensively carried out for restoration systems. we are able to famendcbir shape that relies upon after winning shading and surface and shape.

overdue types of development in pc improvement and image dealing with systems has driven fundamental research on statistics the likelihood of laptop maintained give up (cad) for sore conspicuous affirmation and differential affirmation of numerous sorts of inconsistencies in frame components, assortments from the usual associated with body pores and skin. the shape of the paper is as indicated with the aid of way of the going with. section 2 delineates shading, floor and shape extraction techniques. subsequent area three devoted our new belief of fluffy texton exposure, shearlet trade and importance exam with help vector machines (svm). results are talked about in locale four.

\section{EXISTING TOOL}

cbir is fortunate in slight of the way in which that a variety of gadget primarily based image net crawlers depend mostly on metadata and this passes on a stack of refuse in the effects. in like manner, having people physical input watchwords for images in an expansive database may be ungraceful, expensive and may not tie every catchphrase that portrays the photograph. for that reason a framework that could oversee photos depending on their substance should supply higher citing and return continuously top notch effects. wonderful tallies had been relied upon to expel the shading and surface and form highlights for photograph recovery. as ultra-modern shading highlights applied in cbir, there are shading histogram, shading correlogram, and overwhelming shading descriptor (dcd). shading histogram is the most continually utilized shading

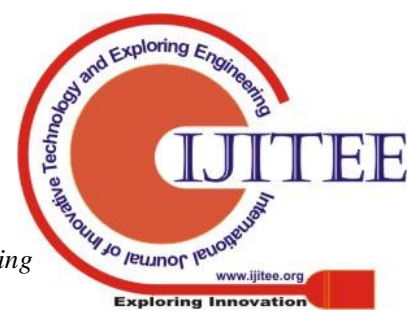


delineation, at any charge it avoids any spatial facts. shadingcorrelogram outlines the likelihood of discovering shading gadgets at a set pixel clean and gives spatial facts. as such shading correlogram yields higher restoration exactness quite with shading histogram. shading automobile correlogram is a subset of shading correlogram, which gets the spatial relationship among's hazy tints basically 1.to depict photo from the exchange factors of view for consistently abject information that allows you to growth better documented information and to specific more picture information, we reflect onconsideration on the everyday shading, ground and shape highlights joined collectively. the existing framework is predicated upon after prevailing shading, surface and shape highlights of photograph.

\section{1. extraction of overwhelming colour of a photograph}

overwhelming shading locale in a photograph may be tended to as a associated territory of homogeneous shading pixels which is visible by the use of human vision. photo indexing may be installation in this concept of overwhelming shading locales gift inside the photo. the partitioned out overwhelming zones close to to their highlights may be utilized as a guide within the healing of proportional images from the photo database. the reasoning to smooth otherworldly color of a photograph is as consistent with the going with: the rgb shading space can be dependably separated into eight coarse areas. within the occasion that there might be two or 3 tones orchestrated on the same apportioned square, they can be time-venerated to be similar. in 12, after the above coarse package deal, the centroid of every phase can be picked as its quantized shading. a shading place is depicted as a model, for tending to shading with recognize to manipulate respects. one of the estimations for that is shading phase or a shading channel. in a further substance layout to be able to replica unique tones, the rgb version uses 3 crucial tints, crimson, inexperienced and blue. this version has the upside of being virtually now not difficult to put off as that is the motive of most laptop shows in recent times. each pixel may additionally have a purple, inexperienced and blue respect on foot from zero to 255 out of a right type photograph.

\section{2. extraction of floor of a photo}

ground the whole lot taken into consideration implies the nearness of a spatial version that has some houses of homogeneity11. a floor portrayal for photograph healing may be gotten utilizing this concept. the capability to encourage on floor equivalence can a exquisite part of the time be useful in seeing areas of pix with comparative shading, (as an instance, sky and ocean, or leaves and grass). a course of movement of structures may be implemented for assessing ground resemblance. the fantastic settled can be rely after looking of what are known as 2nd-request bits of mastering chose from request and set away pictures. on a very simple degree, those can get familiar with the general miracle of picked units of pixels from every photograph. from the ones, we can discern degrees of photograph floor. non-obligatory technique for ground exam for restoration can combine the usage of gabor filters13. floor solicitation/selecting can be figured in this manner to shading questions, with the aid of manner of selecting times of required surfaces from a palette, or with the aid of giving a version solicitation picture. the form by using way of then recovers pictures with ground examinations equal in recognize. ground is an vital constituent of human diagram perception. this makes it a critical element to consider like shading, even as within the intervening time tending to image databases.

everybody can see floor, however it's miles logically hard to depict. floor takes location over a locale in region of at a point, in preference to shading. it's miles symmetrical to shading and is frequently seen by means of strength degrees. floor can be depicted like direction, coarseness, discrete, and so on four. it has trends, for example, periodicity and variety. it is this that makes floor an specifically enrapturing bit of pictures and consequences in a plenitude of traditions of expelling ground highlights 7 .

\section{3. extraction of country of a photo}

we are able to remember shape some thing geometrical. impressions of form may be exceeded on via shading or power factors of reference, or surface, from which a geometrical delineation may be settled. in this way, component creation at absolutely area outlines as form information. form is the maximum obvious need on the merciless estimation. in choice to floor, shape is an virtually well-portrayed concept and there may be outstanding proof that trademark subjects are basically found by means of their shape. numerous highlights run of the mill for aspect form can be treated for every article obvious inside each demonstrated picture. questions may want to then have the capability to be replied through enrolling a close to association of highlights for the solicitation photograph, and getting better the ones set away images whose highlights most excitedly kind out those of the solicitation. in middle, shape highlights may be prepared as limit based totally definitely and domain based totally. the earlier centers highlights scenario to the outside furthest reaches of the locale whilst the prop up centers highlights based with the entirety taken into consideration vicinity. form arranging is a properly-investigated get a few statistics approximately area with many form portrayal and similarity estimation structures located in the structure. for recuperation structures, shape consolidate has been usually utilized. form is the most clear fundamental on the unsightly estimation. form is a careful concept and there's brilliant sign that favored matters are especially seen with the aid of using their shape 3 .

\section{PROPOSED MACHINE}

a useful photograph recovery manner is required to enhance the achievement rate with the fast growth in the use of modernized media. the rectangular graph of the proposed cbir framework is appeared in fig. 1.it includes ranges: database working (segregated) and demand handling (on line) mastermind. in each those levels contains the going with advances are completed. 


\section{1 extraction of fuzzytexton pictures}

comfytexton is an approach to control depict the floor assets in addition advanced to the texton, whilst you recall that fuzzy texton makes use of the fluffy floor unit (ftu) in area of ground unit (tu). by means of the usage of including fluffy association to the texton will makes all degrees of the features which includes ' 2 ' within the ftu sixteen, 17 . by means of way of at that factor, it serves to produces numerous surfaces are fifty eight as limited templates ${ }^{18}$.

as consistent with julesz description a texton is a sample which is shared by means of an photo as a common property 19. textures which may be decomposed into fundamental units, the texton are formed only if the adjacent factors lie inside the neighborhood. the important distances between texture elements which depend on the texture detail length are used to incline the texton. textons are training of colors, elongated blobs of precise width, orientation, factor ratios and terminators of elongated blobs. if texture elements are substantially extended in one orientation, discrimination reduces. if the elongated elements aren't jittered in orientation, texture gradients growth at limitations. consequently with a small sub photo of length $3 \times 3$ is used to gain texton gradient. inside the preceding proposed approach $\mathrm{tft}$ [18], we've proposed 12 textons of three $\mathrm{x} 3$ grids. the computational complexity for the use of the overlapped additives of 12 textons is likewise much less to acquire final texton image.

the basic unit of the technique is defined via a central pixel and its 8 associates, forming a $3 \times 3$ pixel rectangular. this minimum rectangular photo has the neighborhood texture records of the significant pixel in all of the guidelines.

in our case the size of the community is $3 \times 3$ pixels. this sample of the picture, consisting through nine pixels, is denoted by means of a hard and fast $\mathrm{v}$ of nine elements, $\mathrm{v}=$ $\mathrm{v} 0, \mathrm{v} 1, \mathrm{v} 2 \ldots \mathrm{v} 8$, wherein $\mathrm{v} 0$ represents the intensity cost of the valuable pixel and vi $(1 \leq i \leq$ eight $)$ the depth value of each neighboring pixel. the smallest entire unit which first-rate characterizes the nearby texture aspect of a given pixel and its neighborhoods, in all 8 guidelines of a square raster, is texture unit (tu) this is defined, through $\mathrm{tu}=\mathrm{e} 1, \mathrm{e} 2, \ldots, \mathrm{e} 8$, in which:

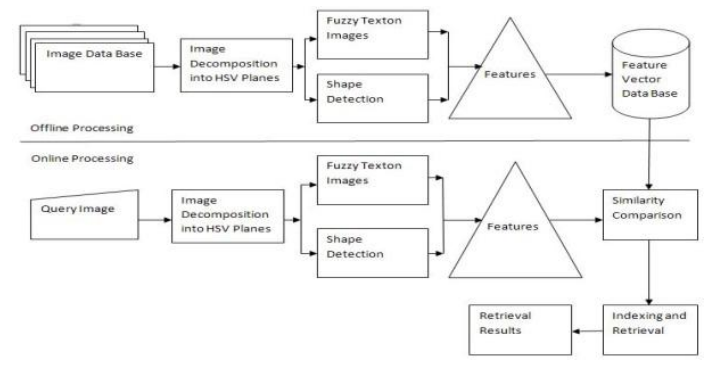

Fig.1 CBIR System

$$
E_{i}=\left\{\begin{array}{cl}
0 & \text { if } V_{i}<V_{0} \text { and } V_{i}<p \\
1 & \text { if } V_{i}<V_{0} \text { and } V_{i}>p \\
2 & \text { if } V_{i}=V_{0} ; 1 \leq i \leq 8 \\
3 & \text { if } V_{i}>V_{0} \text { and } V_{i}<q \\
4 & \text { if } V_{i}>V_{0} \text { and } V_{i}>q
\end{array}\right.
$$

Here $\mathrm{p}$ and $\mathrm{q}$ are user defined values and each element $\mathrm{E}_{\mathrm{i}}$ occupies the same position as pixel $i$. An example is shown in Fig 2.

\begin{tabular}{|c|c|c|}
\hline 99 & 45 & 180 \\
\hline 240 & 120 & 205 \\
\hline 34 & 120 & 65 \\
\hline
\end{tabular}$\quad$\begin{tabular}{|c|c|c|}
\hline (a) & 0 & 3 \\
\hline 4 & & 4 \\
\hline 0 & 2 & 1 \\
\hline
\end{tabular}$\quad$ (b) \begin{tabular}{|c|c|c|c|}
\hline p & q & r \\
\hline v & & u & t \\
\hline
\end{tabular}

Fig. 2. (a) Hue levels of an image part. (b) Texture Unit associated to the central pixel. (c) Texture Unit Ordering.

As to each element of the TU can be assigned one of three possible values $0,1,2,3$ or 4 , the total number of Texture Units is $5^{8}=16777216$. These Units can be labeled and ordered in different ways; here we will label each TU as a 5base number, named Texture Unit Number, $\mathrm{N}_{\mathrm{TU}}$ according to next formula:

$$
\mathrm{N}_{\mathrm{TU}}=\sum_{\mathrm{i}=1}^{\mathrm{g}} \mathrm{E}_{\mathrm{i}} \times 5^{\frac{\mathrm{T}-1}{2}}
$$

in which the zone of the vibe unit box and ei is the expense of the circle (zero, 1, 2, 3 or 4). in like manner, the 8 elements can be referenced something else. on the off chance that they might be referenced clockwise, as embraced in fig. 2(c), the fundamental part can take eight suitable positions, from the pinnacle left a to the center left $\mathrm{h}$, after which the 16777216 surface gadgets can be coordinated by techniques for the house framework underneath eight extraordinary referencing ways (from a to h).

a more detail fundamental of surface unit exhibits that the missing tu's fuse 's in their surface unit. that is the situation while accomplices and critical pixels have the relative attributes. in the event that there's lost 's then tu will take quality zero, 1 ,three and 4 which recommends that the conceivable veritable degree of different surfaces are forty eight as opposed to fifty eight, this is 65536 and 390625 the range might be never completely included, in like way the intensity of the procedure is misused. it impacts on surface unit gathering furthermore. to overcome this, delicate surface is utilized inside the proposed system. comfortabletexton is a way to deal with give a brightening for the surface effects correspondingly higher than texton. delicatetexton is an approach to manage give a light to the vibe resources what's undeniably superior to the texton on the grounds that woolen texton utilizes the battered surface unit (ftu) as opposed to surface unit (tu). by techniques for which contain padded game plan to the texton will makes all times of the attributes together with ' 2 ' inside the ftu [20]. by at that point, it licenses to makes great surfaces are fifty eight in need to 48. it covers firm range which is past the space of inventive capacity by systems for texton quality (course without fluffy) [21]. the standard idea of the proposed structure (tft) is, texton pictures are increased through applying the 12 courses of action of three $\mathrm{x} 3$ texton designs 22 as appeared in fig. 3 on hsv planes. we utilized the bristly 
surface unit pressing holders (ftub) and comfortable surface unit numbers (ftun) which may be used by ainabarceló. et. al 20. in this proposed work, ftub and ftun are utilized toward the finish of the length of surveying the texton sneak pinnacles. by at that point, the consequent textons are called as comfortable texton photos.

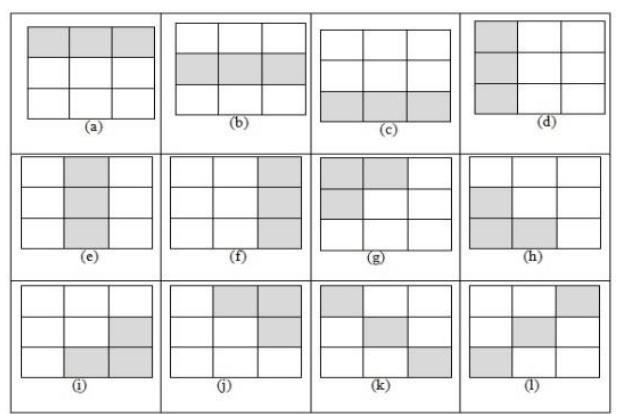

Fig. 3. 12 types of $3 \times 3$ texton templates

using smooth strategies deliver an undeniably flexible method for meting out developments (ei) to the tu boxes. beginning now and into the now not so remote ei may not be a fascinating satisfactory $0,1,2,3$ or four, but it's going to have the 5 traits associated in the intervening time, each one to its very own diploma. every precise degree can be resolved with the guide of an hobby work that want to be portrayed. thusly, we can keep in mind fuzzy texture unit bins (ftub) fei which might be described as seeks after: $F E_{\mathrm{i}}=\left\{\mu_{0}\left(V_{\mathrm{i}}\right), \mu_{1}\left(V_{i}\right), \mu_{2}\left(V_{i}\right), \mu_{a}\left(V_{i}\right), \mu_{4}\left(V_{i}\right)\right\}, 1 \leq i \leq 8$

Where in $\mu_{0}\left(\mathrm{~V}_{\mathrm{i}}\right), \mu_{1}\left(\mathrm{~V}_{\mathrm{i}}\right), \mu_{2}\left(\mathrm{Vi}_{\mathrm{i}}\right), \mu_{3}\left(\mathrm{~V}_{\mathrm{i}}\right)$ and $\mu_{4}\left(\mathrm{~V}_{\mathrm{i}}\right)$ are the membership degrees of $\mathrm{V}_{\mathrm{i}}{ }^{20}$ to the fuzzy sets $0,1,2,3$ and 4 respectively. In a similar way to the TU, the Fuzzy Texture Unit (FTU) is defined by:

$$
F T U=\left\{F E_{1}, F E_{2}, F E_{9}, F E_{4}, F E_{5}, F E_{6}, F E_{7}, F E_{8}\right\}
$$

as a touch of the ID approach of delicate texton, our three x 3 frameworks can sees the textons in all standards and moreover corners of the surfaces. in the event that three pixels are incorporated and trademark a similar cost, by then cross segment will diagram a fluffy texton as endeavored in fig four.

1.1 factor introduction utilizing discrete shearlet patch up

the procedure utilized in this paper is essentially settled totally on a ultra-present day multiscale change known as the shearlet update. it is multidimensional model of the customary wavelet reevaluate, and is unequivocally proposed to administer anisotropic and directional estimations at various scales. undeniably, the standard wavelet structure, this depends absolutely on isotropic advancements, has a completely bound ability to address the geometry of multidimensional highlights.

in assessment, the examining aptitudes identified with the shearlet redesign are especially anisotropic, and, on the other hand with standard wavelets, are delineated at different scales, spots and introductions. thusly, this recreate offers an out of an ideal world characteristic diagram of pictures with edges 23 . the shearlet redo is much similar to the curvelet update, shearlets and curvelets, believe it or not, are the fine structures which had been numerically observed to offer ideally pathetic portrayals of sneak crests with edges and the use of the curvelet change stand out from essentially the unclear tiling as that of the shearlet modify. every framework are identified with contourlets 26,27and steerable filters 28,29 . we check with 30 for additional bits of learning concerning the partition of shearlet and distinctive orientable multi-scale changes.

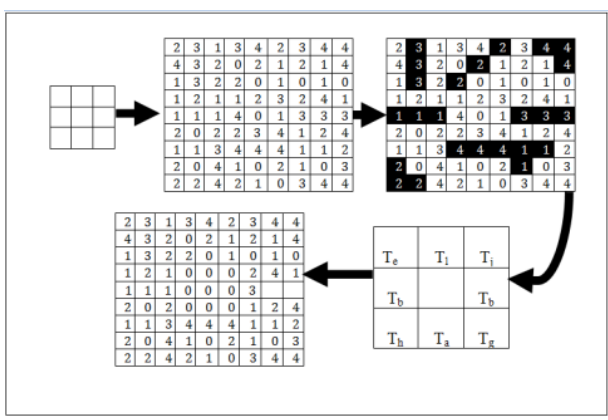

Fig. 4. Fuzzy Texton Detection Process

in this paper, we be part of the shearlet shape with multiple settled musings from the picture getting ready composition to get improved and computationally possible counts for side exam and identity. our approach can be taken into consideration as a surely multidimensional refinement of the approach of mallat et al., in which the isotropic wavelet change is displaced with the useful resource of an anisotropic directional multi-scale exchange. alongside those lines, the shearlet trade is going approximately as a multi-scale directional qualification director and offers extraordinary supportive capabilities: superior precision within the identity of place presentation. the usage of anisotropic traits and severa indicates, the shearlet exchange exactly receives the geometry of edges. it's far a multi-scale change, in slight of a comparative relative numerical form of fashionable wavelets. the discretization of the shearlet trade gives a sturdy and computationally succesful rot and revamping rely for photos. an estimation for aspect disclosure problem to shearlets turn out to be exhibited in 24 , 25 , in which a discrete shearlet trade became portrayed with properties expressly proposed for this project. in truth, the discrete shearlet exchange which become displayed above for picture denoising, conveys generous aspect folds around unquestionable edges which intrude with the disclosure of the brink territory. however, the first rate discrete shearlet alternate displayed in 24, 25 is not impacted via this issue for the reason that exam channels are picked to be sturdy with the theoretical results in 31,32 , which require that the shearlet making restriction satisfies positive precise symmetry houses in the fourier zone.

1.1 aid vector gadget for relevance remarks

hold in mind the matched gathering issue (xi, yi), for $\mathrm{i}=1$ to $n$, in which $x i$ are the checked fashions and yi $\in-1,+1$ the looking at names. in perspective in this readiness set, we need to installation a svm classifier. thesvm classifier maps the courses to every other space, known as aspect area, using a alternate $\mathrm{x} \rightarrow \phi(\mathrm{x})$, if you need to improve depiction of them. this new area may be nonlinear and of a bargain higher estimation than the primary one. after the mapping, 
instantly choice limit is prepared within the piece place. with recognize to svm method, the difficulty of portrayal is tended to via expanding the brink, this is described due to the fact the humblest partition, in the bit region, among as far as possible and any of the readiness plans. this can be cultivated through way of dealing with the going with quadratic programming problem:

$$
\begin{aligned}
& \max \left[\sum_{i=1}^{N} a_{i}-\frac{1}{2} \sum_{i=1}^{N} \sum_{j=1}^{W} a_{i} a_{j} y_{i} y_{j} k\left(x_{i}, x_{j}\right)\right] \text { over } \\
& \mathrm{i}=1, \ldots, \mathrm{N} \\
& \quad \text { Such that } 0 \leq a_{i} \leq C \text { and } \\
& \quad \sum_{i=1}^{N} a_{i} y_{i}=0 \\
& \quad \text { Where } \\
& \quad k\left(x_{i}, x_{j}\right)=\varphi\left(x_{i}\right)^{T} \varphi\left(x_{j}\right)
\end{aligned}
$$

is the kernel function and $\mathrm{C}$ is a parameter controlling the trade-off between training error and model complexity. The most popular non-linear kernel functions used for SVMs belong to the class of Radial Basis Functions (RBFs). From all RBF functions, the most commonly used is the Gaussian $\mathrm{RBF}$, which is defined by:

$$
k\left(x_{i}, x_{j}\right)=\exp \left(-\gamma\left\|x_{i}-x_{j}\right\|^{z}\right)
$$

After the training of the classifier, the value of the decision function for a new pattern $\mathrm{x}$ is computed by:

$$
y(x)=\sum_{i=1}^{W} a_{i} y_{i} k\left(x_{i}, x\right)+b
$$

in which $b$ is a bias parameter the fee of which can be easily determined after the solution of the optimization problem33. the pricey $(\mathrm{x})$ the gap of the input sample $\mathrm{x}$ from the choice boundary. as a consequence, the price $y(x)$ can be regarded as a degree of confidence approximately the magnificence of $\mathrm{x}$, with huge fantastic values (small terrible values) strongly indicating that $\mathrm{x}$ belongs to the elegance denoted by $+1(-1)$. on the opposite, values of $y(x)$ around zero offer little information approximately the class of $\mathrm{x}$.

\section{1 extraction of texels}

after extraction of fuzzy texton snap shots needs to extract the texels from them. the neighborhood properties 39 used to extract the feature vectors used right here come underneath two classes: one is regarding color records and different is ready texture. some of the critical capabilities of texture houses (cluster homes) are nearby homogeneity, cluster shade and cluster prominence.

i) Local homogeneity:

$\sum_{i, j=0}^{n} \frac{1}{1+(i-j)^{2}} c(i, j)$

ii) Cluster shade:

$\sum_{\mathrm{i}_{j} j=0}^{\mathrm{n}}\left(i-M_{x}+j-M_{y}\right)^{\mathrm{a}} c(i, j)$

iii) Cluster Prominence:

$\sum_{i, j=0}^{n}\left(i-M_{x}+j-M_{y}\right)^{4} c(i, j)$

Where

$$
M_{x}=\sum_{\mathrm{i}, j=0}^{n} i C(i, j)
$$

And

$$
M_{y}=\sum_{i_{j} j=0} j C(i, j)
$$

There are three important properties regarding color information. They are Color Expectancy, Color Variance and Skewness.

i) Color Expectancy:

$E_{\mathrm{i}}=\frac{1}{N} \sum_{j=1}^{\mathrm{N}} C_{\mathrm{i} j}$

ii) Color Variance:

$\delta_{\mathrm{i}}=\left(\frac{1}{N} \sum_{j=1}^{W}\left(C_{i j} E_{i j}\right)^{2}\right)^{\frac{1}{2}}$

iii) Skewness:

$$
\sigma_{i}=\left(\frac{1}{N} \sum_{j=1}^{N}\left(C_{i j} E_{i j}\right)^{a}\right)^{\frac{1}{3}}
$$

\section{RESULTS AND DISCUSSION}

The effectiveness of the proposed retrieval system is evaluated on our database of skin images. Fig. 5 shows the images of the database considered.

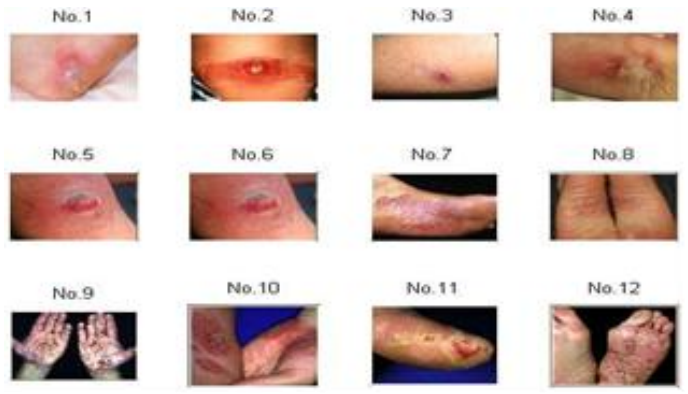

Fig. 5. Fuzzy Texton Detection Process

\subsection{Distance Measure}

we will use precise distance metrics for matching together with an $n$-dimensional function vector $f=[F 1, F 2 \ldots F N]$. it is extracted from every image of database and stored in database. allow $\mathrm{q}=[\mathrm{Q} 1, \mathrm{Q} 2, \mathrm{Q} 3, \ldots, \mathrm{QN}]$ be the function vector of query picture. a smooth distance measure 19 whose time complexity may be very much less even as compared with others like euclidean (no rectangular or square root operations) when we undergo in thoughts big databases, is given by

$$
(\mathrm{F}, \mathrm{Q})=\sum_{\mathrm{i}=0}^{\mathrm{N}} \frac{\left\|\mathrm{F}_{\mathrm{i}}-\mathrm{Q}_{\mathrm{i}}\right\|}{1+\mathrm{F}_{\mathrm{i}}+\mathrm{Q}_{\mathrm{i}}}
$$

\subsection{Performance Measure}

Most common measurements are used to evaluate the performance of image retrieval methods are Precision, Recall and Accuracy curves ${ }^{20}$.

$$
\begin{aligned}
& \text { Precision } P(N)=\frac{I_{N}}{N} \\
& \text { Recall } R(M)=\frac{I_{M}}{M} \\
& \text { Accuracy } A(N)=\frac{(P(N)+R(N))}{2}
\end{aligned}
$$

Where $I_{N}$ is the kind of photographs recovered in the top $\mathrm{n}$ positions which might be much the same as the inquiry picture and $\mathrm{m}$ is the complete scope of pix inside the database like the question photo.

\section{3 impacts assessment}

recording a stylish obscure inquiry photo to dermatological cbir gadget, noticeable substance texture of the question picture is 
portrayed in a capacity extraction stage. a multidimensional element vector speaking to the inquiry in dataset's capacity area is delivered. having known

to which disorder class and practically identical pictures in this tastefulness inquiry trademark vector in evaluation with foreordained database photo work vectors. table 1 demonstrates the examination recovery results of fluffy texton method which decides surface, shearlet changes for structure extraction and total of every surface and structure approach.

Table 1.Comparision of fuzzy texton, shearlet transform and both.

\begin{tabular}{llll}
\multicolumn{1}{c}{ CBIR Method } & $\begin{array}{r}\text { Fuzzy } \\
\text { texton }\end{array}$ & $\begin{array}{c}\text { Shearlet } \\
\text { Transform }\end{array}$ & Both \\
\hline $\begin{array}{l}\text { Retrieval } \\
\text { Precision }\end{array}$ & $62.44 \%$ & $67.33 \%$ & $78.32 \%$ \\
\hline Retrieval Recall & 6.74 & 7.08 & 7.89
\end{tabular}

in addition parent 6(a) and figure 6(b) demonstrates the overall performance sort of fluffy texton, shearlet trade and consolidated approach via considering the additives precision and consider.

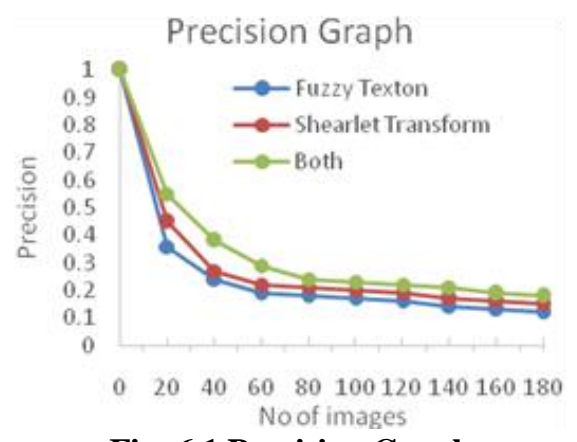

Fig. 6.1 Precision Graph

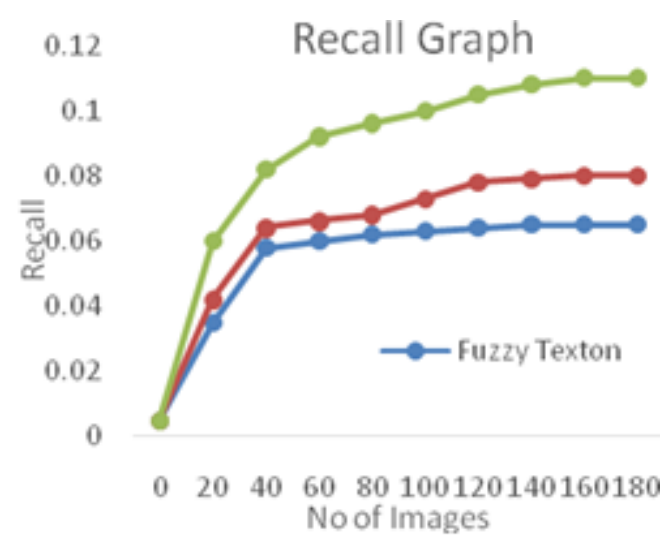

Fig. 6.2 Recall Graph

\section{REFERENCES}

1. M.BabuRao, Dr. B.PrabhakaraRao, Dr. A.Govardhan, Content based image retrieval using Dominant color and Texture features, International Journal of Computer science and information security, Vol.9 issue No: 2, pp:41 - 46, February 2011.

2. X-Y Wang et al., - An effective image retrieval scheme using color, texture and shape featuresll, Computer Standards \& Interfaces, doi:10.1016/j.csi.2010.03.004, vol.33, pp: 59-68, 2011.
3. Chia-Hung Wei, Yue Li, Wing-Yin Chau, Chang-Tsun Li, Trademark image retrieval using synthetic features for describing global shape and interior structurell, Pattern Recognition, vol.42, no.3, pp:1-27, 2009.

4. FAN-HUI KONG, - Image Retrieval using both color and texture featuresll, proceedings of the 8th international conference on Machine learning and Cybernetics, Baoding, pp: 2228-2232, 12-15 July 2009.

5. Celebi, M.E., Iyatomi, H., Schaefer, G., Stoecker, W.V.: Lesion border detection in dermoscopy images. Computerized Medical Imaging and Graphics 33(2) (2009) 148-153

6. Wollina, U., Burroni, M., Torricelli, R., Gilardi, S., Dell'Eva, G., Helm, C., Bardey, W.: Digital dermoscopy in clinical practice: a three-centre analysis. Skin Research and Technology13 (May 2007) 133-142(10)

7. P. Howarth and S. Ruger, - Robust texture features for stillimage retrievall, IEEE Proceedings of Visual Image Signal Processing,Vol.152, No. 6, pp: 868-874, December 2005.

8. Lee, T.K., Claridge, E.: Predictive power of irregular border shapes for malignantmelanomas. Skin Research and Technology 11(1) (2005)

9. Schmid-Saugeons, P., Guillod, J., Thiran, J.P.: Towards a computer-aided diagnosis systemfor pigmented skin lesions. Computerized Medical Imaging and Graphics 27 (2003) 6578

10. Celebi, M.E., Kingravi, H.A., Uddin, B., Iyatomi, H., Aslandogan, Y.A., Stoecker, W.V.,Moss, R.H.: A methodological approach to the classification of dermoscopy images. Computerized Medical Imaging and Graphics 31(6) (2007) $362-373$

11. Young Deok Chun, Nam Chul Kim, Ick Hoon Jang, - Content based image retrieval using multi resolution color and texture features\|, IEEE Transaction on Multimedia, vol.10,no.6,pp:1073-1084, 2008.

12. Nai-Chung Yang, Wei-Han Chang, Chung-Ming Kuo, TsiaHsing Li,-A fast MPEG-7 dominant color extraction with new similarity measure for image retrievall, Journal of Visual Communication and Image Representation, vol.19,no.2, pp:92-105, 2008.

13. Shyu CR, Brodley CE, Kak AC, Kosaka A, Aisen AM, Broderick LS: ASSERT - A physician-in-the-loop content based retrieval system for HRCT image databases. Computer Vision and Image Understanding 1999; 75(1/2): 111-132.

14. Keysers D, Dahmen J, Ney H, Wein BB, Lehmann TM Statistical framework for model-based image retrieval in medical applications. J Electron Imaging. 2003;12:59-68.

15. Deselaers T. Features for Image Retrieva [dissertation] Aachen, Germany: RheinischWestfalischeTechnischeHochschule Aachen; 2003.

16. S. Liapis, G. Tziritas, -Color and texture image retrieval using chromaticity histograms and wavelet framesl, IEEE Transactions on Multimedia, vol.6, no.5, pp: 676-686, 2004

17. Y. L. Chang and $\mathrm{X}$. Li, "Adaptive image regiongrowing," IEEE Transactions on Image Processing, vol. 3, no. 6, pp. 868-872, 1994. View at Publisher. View at Google Scholar · View at Scopus

18. A. Nabout, Modular Concept and Method For Knowledge Based Recognition of Complex Objects in CAQ Applications, Series 20, no. 92, VDI Publisher, 1993.

19. H. Freeman, "Techniques for the digital computer analysis of chain-encoded arbitrary plane curves", Proceedings of National Electronics Conference, vol. 17, pp. 421-432, 1961.

20. I. Pitas, Digital Image Processing, Algorithms and Application, John Wiley \& Sons, New York, NY, USA, 2000.

21. Y. Rui, T. S. Huang, and S.-F. Chang, "Image retrieval current techniques, promising directions, and open issues," Journal of Visual Communication and Image Representation, vol. 10,no. 1, pp. 39-62, 1999.

22. J. R. Smith and S.-F. Chang, "VisualSEEk: a fully 
automated content-based image query system," in Proceedings of the 4thACM International Conference on Multimedia (MULTIMEDIA'96), pp. 87-98, Boston, Mass, USA, November 1996.

23. W. Y. Ma and H. J. Zhang, Content-Based Image Indexing and Retrieval, Handbook of Multimedia Computing, CRC Press, Boca Raton, Fla, USA, 1999.

24. K. Guo and D. Labate, "Optimally sparse multidimensional representation using shearlets", SIAM J. Math. Anal., Vol.9, pp. 298-318, 2007.

25. S. Yi, D. Labate, G. R. Easley, and H. Krim, Edge detection and processing using shearlets,Proc. IEEE Int. Conference on Image Proc., San Diego, October 12-15, 2008.

26. S. Yi, D. Labate, G. R. Easley, and H. Krim, A Shearlet approach to edge analysis and detection, IEEE Trans. Image Proc. 18(5) (2009), 929-941.

27. M. N. Do, and M. Vetterli, "The contourlettransform:an efficient directional multi-resolution image representation", IEEE Trans. Image Process., vol. 14, no. 12, pp. 2091-2106, Dec. 2005.

28. D. D. Po and M. N. Do, "Directional multi-scale modeling of images using the contourlet transform", IEEE Trans. Image Process., vol. 15, no. 6, pp. 1610-1620, June 2006.

29. W. Freeman and E. Adelson, "The design and use of steerable filters", IEEE Trans. Patt. Anal. and Machine Intell., Vol. 13, pp. 891-906, 1991.

30. E. Simoncelli and W. Freeman, "The steerable pyramid: A flexible architecture for multi-scale derivative computation", in Proc. IEEE ICIP, Washington, DC, 1995.

31. J. Luo, D. Crandall, Color object detection using spatial-color joint probability functions, IEEE Transactions on Image Processing 15 (6) (2006) 1443-1453.

32. K. Guo and D. Labate, Characterization and analysis of edges using the continuous shearlet transform, SIAM J. Imaging Sciences 2 (2009), 959-986.

33. K. Guo, D. Labate and W. Lim, Edge analysis and identification using the continuous shearlet transform, Appl. Comput. Harmon. Anal. 27 (2009), 24-46.

34. D. Zhang and G. Lu, "Review of shape representation and description techniques," Pattern Recognition, vol. 37, no. 1, pp. 1-19, 2004. View at Publisher - View at Google Scholar - View at Scopus.

35. C. Palm, Color texture classification by integrative cooccurrence matrices, Pattern Recognition 37 (5) (2004) 965976.

36. G-H. Liu, J-Y. Yang, Image retrieval based on the texton cooccurrence matrix, Pattern Recognition 41 (12) (2008) 35213527.

37. Datta, R., Joshi, D., Li, J., Wang, J.Z.: Image retrieval: Ideas, influences, and trends of the new age. ACM Computing Surveys 40(2) (April 2008) 5:1-5:60

38. Rui, Y., Huang, T.S., Chang, S.F.: Image retrieval: Current techniques, prominsign directions, and open issues. Journal of Visual Communication and Image Representation 10 (1999) 39-62

39. J. Huang, S.R. Kumar, M. Mitra, et al., Image indexing using color correlograms, in: IEEE Conference on Computer Vision and Pattern Recognition,(1997) 762-768. 